\title{
BMJ Open Senior medical students as assistants in medicine in COVID-19 crisis: a realist evaluation protocol
}

\author{
Lynn V Monrouxe (D) , ${ }^{1}$ Peter Hockey, ${ }^{1,2}$ Priya Khanna, ${ }^{3}$ Christiane Klinner, ${ }^{1}$ \\ Lise Mogensen, ${ }^{4}$ Deborah A O'Mara, ${ }^{3}$ Abbey Roach, ${ }^{2}$ Stephen Tobin, ${ }^{4}$ \\ Jennifer Ann Davids (iD ${ }^{2}$
}

\begin{abstract}
To cite: Monrouxe LV, Hockey P, Khanna P, et al. Senior medical students as assistants in medicine in COVID-19 crisis: a realist evaluation protocol. BMJ Open 2021;11:e045822. doi:10.1136/ bmjopen-2020-045822

- Prepublication history for this paper is available online. To view these files, please visit the journal online (http://dx.doi org/10.1136/bmjopen-2020045822).
\end{abstract}

Received 15 0ctober 2020 Accepted 21 July 2021

Check for updates

(C) Author(s) (or their employer(s)) 2021. Re-use permitted under CC BY-NC. No commercial re-use. See rights and permissions. Published by BMJ.

${ }^{1}$ Faculty of Medicine and Health, The University of Sydney,

Sydney, New South Wales, Australia

${ }^{2}$ Research and Education Network, Western Sydney Local Health District, Sydney, New

South Wales, Australia

${ }^{3}$ Sydney Medical School, The University of Sydney, Sydney, New South Wales, Australia

${ }^{4}$ School of Medicine, Western

Sydney University, Penrith South, New South Wales, Australia

Correspondence to Dr Jennifer Ann Davids; jennifer.davids@health.nsw. gov.au

\section{ABSTRACT}

Introduction The assistant in medicine is a new and paid role for final-year medical students that has been established in New South Wales, Australia, as part of the surge workforce management response to the COVID-19 pandemic. Eligibility requires the applicant to be a finalyear medical student in an Australian Medical Councilaccredited university and registered with the Australian Health Practitioner Regulation Agency. While there are roles with some similarities to the assistant in medicine role, such as assistantships (the UK) and physician assistants adopted internationally, this is completely new in Australia. Little is known about the functionality and success factors of this role within the health practitioner landscape, particularly within the context of the COVID-19 pandemic. Given the complexity of this role, a realist approach to evaluation has been undertaken as described in this protocol, which sets out a study design spanning from August 2020 to June 2021.

Methods and analysis The intention of conducting a realist review is to identify the circumstances and mechanisms that determine the outcomes of the assistant in medicine intervention. We will start by developing an initial programme theory to explore the potential function of the assistant in medicine role through realist syntheses of critically appraised summaries of existing literature using relevant databases and journals. Other data sources such as interviews and surveys with key stakeholders will contribute to the refinements of the programme theory. Using this method, we will develop a set of hypotheses on how and why the Australian assistants in medicine intervention might 'work' to achieve a variety of outcomes based on examples of related international interventions. These hypotheses will be tested against the qualitative and quantitative evidence gathered from all relevant stakeholders.

Ethics and dissemination Ethics approval for the larger study was obtained from the Western Sydney Local Health District (2020/ETH01745). The findings of this review will provide useful information for hospital managers, academics and policymakers, who can apply the findings in their context when deciding how to implement and support the introduction of assistants in medicine into the health system. We will publish our findings in reports to policymakers, peer-reviewed journals and international conferences.
Strengths and limitations of this study

- A realist evaluation design is able to provide a deeper level of understanding as to how an intervention in complex situation works by assessing the interaction of the underlying causal factors through an investigation of the context, mechanisms and outcomes.

- The ongoing and iterative nature of the realist syntheses and evaluation allows for complex interpretation of the programme theory and the development of middle-range theories.

- The survey will be sent to 55 assistant in medicine participants at the end of the programme, which may prove problematic in terms of data analysis.

\section{INTRODUCTION}

New South Wales (NSW) State Ministry of Health, the largest health system in Australia, reviewed its workforce capacity in anticipation of a COVID-19 surge. As a result, a new assistant in medicine role has been created to work within non-COVID-19 multidisciplinary teams to provide extra medical assistance should junior medical officers be redeployed. These roles have been filled by final-year medical students. These students volunteered via their medical school, which reviewed their progress and certified them as having appropriate knowledge and skills. The assistant in medicine role is officially paid, workplace employment, rather than a clinical placement. Despite students being able to express an interest in a particular placement, this role has been designed so that it aligns with local health district service needs rather than with the students' potential career interests. The role is part-time and based on a temporary contract extending to a maximum of 6 months at 32 hours per week, with variation across different local health districts, which equates to approximately 3-4 shifts per week at the most. The assistants in medicine 
continued with their university medical course 1 day per week to fulfil their curriculum requirements. Furthermore, some assistants in medicine received synchronous and asynchronous educational sessions and engaged with entrustable professional activities (a competency framework). ${ }^{1}$ This unique assistant in medicine initiative has provided an opportunity to evaluate whether the clearly defined expectations and intended outcomes desired by the various stakeholders (including universities, local health districts and the NSW State Ministry of Health) are met. The results from the study will be disseminated to these key stakeholders to inform future policy decisionmaking concerning the ongoing nature of the assistant in medicine role and inform curriculum designers within medical schools regarding final-year students' preparedness for practice issues. We also anticipate a reciprocal transferability of knowledge with other related initiatives outlined below.

\section{Relationships to other initiatives}

There are a number of existing roles that relate to the assistant in medicine scheme. These include the roles of assistantships in medicine,${ }^{2-6}$ introduced in the UK to ease the transition of final-year medical students into their junior doctor roles, and the physician assistant/physician associate $^{7-20}$ role, first introduced in the USA. Although these roles differ from the assistant in medicine role, they do offer significant insights into the implications of introducing new roles to fill the vacancy of clinical personnel within the hospital system and considering new options for the transition into practice for final-year medical students. We outline these roles below.

\section{Medical students' assistantships}

Assistantships in the UK are medical students who, via a longitudinal full-time placement, are integrated into a healthcare team for the last few months of their clinical training to gain phased-in hands-on experience carrying out the work of a newly qualified doctor under appropriate supervision. ${ }^{2-521}$ Thus, the timing of assistantships is the same as the assistants in medicine. Similar to assistants in medicine, assistantships differ in length from 3 to 6 months, and they can be undertaken in the hospital where students will eventually be appointed. ${ }^{56}$ However, unlike assistants in medicine, some assistantships are aligned with the exact role to which they are about to transition. ${ }^{56}$ The purpose of this assistantship role is to smooth the transition from being a student to being a professional, ${ }^{22}$ hopefully easing their passage into a professional role by gradually preparing them for the responsibilities they will face as a junior doctor. ${ }^{22}{ }^{24}$ This purpose is only partly aligned with that of the assistants in medicine, as their key purpose was to provide assistance should junior medical officers be otherwise deployed (so they are there to fill a service gap due to the pandemicrelated demands).

This assistantship role is not without its challenges. Some students report struggling to participate effectively due to a lack of clarity about the nature of their role. Consequently, students narrate becoming passive, preventing open participation, active learning and the development of professional identity as a junior doctor. ${ }^{2}$ This, it is suggested, can compound student stress and clinical risk, and hamper meaningful appraisal of their professional development. ${ }^{224}$ As a result, the development of a sense of belonging and feeling like a doctor (ie, their professional identity) may be delayed due to the lack of meaningful participation in professional activities. ${ }^{2}$

Students who report being supported narrated their experiences in a markedly different manner to those who did not. Indeed, this support eased students' transition into their professional role, feeling it to be 'business as usual' due to their existing understanding of requirements and work practices for an F1 (Post Graduate Year 1(PGY1).${ }^{24}$ As Crossley and Vivekananda-Schmidt state, 'the gap then between student and doctor is quite clear. It is participation in healthcare delivery with a real purpose'. ${ }^{2}$ Indeed, responsibility and participation in professional activities appear to be crucial for furnishing students with confidence, resilience and proactive behaviours in professional practice and reinforcing aspects of personal development also delivered in the university curriculum. ${ }^{24}$ Key aspects of the assistantship programme appear to be the quality of supervision provided to students in this role ${ }^{325}$ and the extent to which they are accepted and mentored into multidisciplinary teams. ${ }^{2}$

The unpaid assistantship differs from the assistant in medicine role in that it is a full-time student role with the main intention of gradually integrating students into practice as paid employees, while the assistant in medicine is a part-time role that was intended to act as a fully functioning team member. They are similar to each other in that both roles are held by final-year medical students at the end of their degree, and there is an element of transition smoothing with both.

\section{Physician assistant/associate}

The role of physician assistant or physician associate was introduced in the USA in 1965 and then developed internationally (eg, in countries such as Australia, Canada, England, the Netherlands, Scotland, South Africa and Taiwan). In Australia, this role was proposed to meet the demand for medical services following a drive for a healthier society through the introduction of Medicare; however, it wasn't implemented. ${ }^{18}$ Physician assistant/ associates are usually able to undertake routine technical tasks and so relieve the load of the physician. ${ }^{26}$ However, physician associates are less qualified than a physician and unable to work independently. ${ }^{18}$

A number of impediments restricting the use of physician assistant/Associates have been identified including legal issues, training programmes, lack of medical school attendance and unclear role relationships. ${ }^{7}$ However, it has been found that the physician assistant/associate role can reduce pressure on struggling health systems and successfully fill a much-needed gap in healthcare. In 
addition, where these roles are deployed, patient feedback is largely favourable, and there is reportedly an increase of team flexibility, continuity of care and smooth patient flow. ${ }^{914}$ Physician assistant/associates offer the capacity to fill roles currently filled by medical staff, thus saving on resources, ${ }^{16}$ and provide opportunities for doctors to spend time on more complex patients and to attend to patients in clinic and theatre settings. ${ }^{14}{ }^{15}$ Physician assistant/associates are found to be valued for their generalism, health background, confidence in differential diagnoses and communication. Furthermore, the presence of physician assistant/associates can enhance postgraduate medical education through filling in for junior doctors and releasing them from duties. ${ }^{12} 13$ However, doctors are concerned about the reduction of medical education opportunities for junior doctors caused by this role as attention is diverted to the training of new physician assistant/associates, which is not generally supported in practice.

In fact, this role has caused great disquiet among junior doctors. For example, in the UK, junior doctors voted to 'actively oppose' the medical associate professionals (MAPs) to being treated equally to them in relation to medical staffing. MAPs include physician associates and advanced critical care practitioners. ${ }^{11}$ Issues associated with the role are based around regulation, registration, autonomy and a lack of understanding or knowledge about the role..$^{9} 151920$ Ignorance about the physician assistant/associate role can cause problems for physician assistant/associates with regard to identity formation and identity dissonance, ${ }^{8}$ and there are issues around managing the expectations of the role by both those training to be physician assistant/associates and healthcare staff. ${ }^{10}$ Lastly, lack of options for physician assistant/ associates can impact career advancement, and there is a propensity for burnout. ${ }^{19}$

While both the physician assistant/associate and assistant in medicine roles are paid positions and both filling a service gap, there are differences between the roles. For example, the physician assistant/associate is full time and does not include integrated study time, and the role will not lead to the position of a physician or open a career pathway to further progression or result in autonomy of practice. Second, the former have graduated from their medical programme, whereas the assistant in medicine has not graduated with some assistants in medicine having more curriculum and assessment to undertake. Furthermore, physician assistant/associates are interdependent, semiautonomous clinicians practising in partnership with physicians, whereas assistants in medicine work under clinicians' supervision. The tasks that each assistant in medicine student is allowed to do are expected to vary according to the hospital and team they are allocated to.

\section{Research aims}

The NSW Health-sponsored Assistant in Medicine Initiative provides a unique opportunity to assess the extent to which this new workforce model works to achieve different outcomes for the stakeholders involved in the initiative (across both educational and workplace settings). This study seeks to evaluate the Assistant in Medicine Initiative by unpacking the nuances using realist synthesis and realist evaluation ${ }^{27-29}$ in an integrated, coordinated and collaborative approach. Given the diverse range of expected outcomes by different stakeholders (namely, clinical schools, local health districts, the Ministry of Health, the assistants in medicine and their team workers), the protocol could be applied to other sites where this role has been implemented.

\section{RESEARCH QUESTIONS (RQS)}

Our study has two overarching RQs:

RQ1: To what extent does the assistant in medicine intervention meet the expectations (outcomes) of stakeholders, in terms of what works, for whom, how and in what circumstances?

RQ2: What conclusions can we draw from our findings that will benefit the future development and implementation of an assistant in medicine-type programme as an ongoing venture for final-year medical students?

\section{METHODS}

Theory-driven approaches such as realist synthesis and realist evaluation will be used to address our RQs. Based on the Realist And Meta-narrative Evidence Syntheses: Evolving Standards (RAMESES) protocol, ${ }^{30}$ the evaluation is based on three phases, namely, phase 1, realist synthesis; phase 2, realist evaluation; and phase 3 , analysis.

Underpinned by realist philosophy of science, the methods' strength lies in providing a generative understanding of causality. Thus, for any specific outcome $(\mathrm{O})$, there are underlying mechanisms $(\mathrm{M})$ that cause that outcome in a given context $(\mathrm{C})$. These underlying mechanisms are not obvious and are subject to the interaction of combining factors that may alter depending on the opportunities that are embedded in specific context(s). An exploration of these mechanisms can reveal the drivers behind (un)intended outcomes and explain the circumstances in which these mechanisms are activated. Below, we outline the phases of our study.

\section{Phase 1: a realist synthesis}

Realist synthesis comprises a broad-based review of all literature available (including electronic articles, books and grey literature) regarding similar initiatives and roles (as outlined above). This study follows the iterative steps suggested by Pawson $e t a l^{28}$ and implemented in previous reviews undertaken by the lead author. ${ }^{31}{ }^{32}$ We plan to report our realist syntheses according to RAMESES publication standards. ${ }^{33}$ This synthesis work will facilitate our development of an initial programme theory in which we will hypothesise the intended outcomes of the programme and the proposed mechanisms that bring forth those outcomes, alongside the various contexts in 
which we believe these to occur. Through testing (see phase 3), this ideally results in a 'revised, more nuanced and more powerful programme theory'. ${ }^{29}{ }^{31}$ The steps through which we will undertake our work are as follows:

\section{Step 1: clarify the scope, locate existing theories and develop} programme theory

We will conduct a broad database scan to search for existing theories, based on our own hypotheses, to help us build our initial programme theory. We will search through electronic published sources. We will identify the variations of the assistant in medicine role such as the physician assistant/associate and the assistantships in medicine and examine how they are supposed to work and their intended outcomes (developing initial C-M-Os). Variations will be considered if they have considerable overlap with the assistant in medicine role in either the rationale for their inception (ie, to fill a physician service gap/need) or they involve final-year medical students learning/working in the clinical setting as they transition into their first job. We will review initial C-M-Os, examining what these programmes achieve and also for explanations as to why such programmes do not always achieve expected outcomes.

Our search of literature to date has identified a number of aspects that might impact on the implementation of the role. Some of these include role regulation, acceptance, integration, extension and support. For example, a significant factor in the quality of the experience of an assistant in medicine is the appropriate delegation of substantial or significant responsibility to them, thereby integrating them into the team. Where this has occurred under appropriate supervision, the assistants in medicine experienced a higher level of professional development and preparedness. This requires an understanding of this role within the hospital system and how it can be effectively used. By comparison, the role of physician assistant has been hampered by a lack of a clear job description ${ }^{20}$ and ability to act with authority, leading to calls for regulation and registration within a new association. ${ }^{17} 1920$ Confusion about these roles can severely impact on the formation of professional identity leading to identity dissonance. $^{28}$
Step 2: search for evidence

Table 1 clarifies our inclusion and exclusion criteria for the literature search. Using these criteria, we will work with a university-based librarian to develop an appropriate search strategy to locate articles pertinent to the roles of assistantships in medicine, physician assistants and physician associates (box 1 is an example of this). Note that we include the term 'pandemic' in our search strategy as the assistant in medicine role was implemented in response to the pandemic. The rationale is to see if any other similar roles have been developed or any equivalent use of senior medical students, during the pandemic, and how they are being used. As the search develops, we will continue to iteratively monitor and assess our search terms, introducing new terms as required. An additional search of grey literature will commence if deemed appropriate, in which we will review documentation that contains policy, procedures and curriculum reports alongside any other literature that may come within the scope of the study.

\section{Step 3: study selection procedure and appraisal}

We will search first for evidence-based peer-reviewed articles and non-peer reviewed forms of literature such as conference papers, reviews and editorials published between 2015 and 2020. A reference list will be created in EndNote of titles and abstracts of the literature identified. As we are undertaking a realist synthesis, we will apply an iterative model of literature review: refining and reviewing theoretical elements as they are formed and developed. Any findings that are significant but stretch the inclusion criteria will be included, and the boundaries of the preliminary inclusion criteria will be adapted accordingly.

\section{Step 4: data extraction and organisation}

Relevant literature will be extracted in an Excel spreadsheet using realist synthesis appraisal form that includes the following categories: author, title, year of publication, construct under study, design, methods and findings. The literature will be graded, as advised by the RAMESES standards, ${ }^{30}$ according to robustness and relevance to the programme theory, and will be checked for integrity and reliability. In this way, we will be able to evaluate the quality

\begin{tabular}{|c|c|}
\hline Construct & Criteria \\
\hline Timespan & $\begin{array}{l}\text { 2015-2020: exclude dates outside this range. Exception: key articles such as similar interventions due to } \\
\text { past pandemics that may be found outside this date range }\end{array}$ \\
\hline Reference types & $\begin{array}{l}\text { Full research papers, editorials, commentaries, brief reports and other short pieces, book chapters and } \\
\text { conference proceedings. Exclude unpublished works }\end{array}$ \\
\hline Research design & All kinds of research design \\
\hline Participant groups & $\begin{array}{l}\text { Final-year medical students, physician associates/assistants and other types of physician assistants } \\
\text { designed to fill a service gap. Exclude all medical and non-medical personnel outside the inclusion range }\end{array}$ \\
\hline Study contexts & $\begin{array}{l}\text { Hospital sites and similar clinical learning environments (eg, ambulatory settings). Exclude all contexts } \\
\text { outside the inclusion range }\end{array}$ \\
\hline Languages & Articles written in English. Exclude other languages \\
\hline
\end{tabular}




\section{Box 1 Example of search strategy}

Ovid Technologies Email Service

Search for: 1 or 18 or 19

Results: 1

Database: MEDLINE(R) including daily update (1996-current) search strategy:

1. One assistantship ${ }^{*} . \mathrm{mp} .(56)$

2. Two physician assistants.mp. or physician assistants/ (4178)

3. (Clinician* adj2 (associate or associates or aide or aides or assistant or assistants)).ti,ab. (74)

4. (Doctor ${ }^{\star}$ adj2 (associate or associates or aide or aides or assistant or assistants)).ti,ab. (150)

5. (Clinical adj1 (associate or associates or aide or aides or assistant or assistants)).ti,ab. (231)

6. (Physician* adj1 (associate or associates or aide or aides or assistant or assistants or extender )).ti,ab. (3107)

7. (Medical ${ }^{*}$ adj1 (associate or associates or aide or aides or assistant or assstants)).ti,ab. (306)

8. Two or three or four or five or six or seven (5504)

9. Pandemics/ (28328)

10. Coronavirus infections/ (28137)

11. COVID-19*.mp. (24457)

12. Severe acute respiratory syndrome/ or SARS virus/ or sars.mp. (16339)

13. Nine or 10 or 11 or 12 (42096)

14. Medical education.mp. or education, medical/ (48897)

15. Education, medical, undergraduate/ (17007)

16. Medical students.mp. or students, medical/ (35915)

17. 14 or 15 or 16 (78809)

18. 13 and $17(310)$

19. Limit eight to $\mathrm{yr}=$ '2015-current' (1898)

20. One or 18 or 19 (2262)

of the research literature and the richness of its conceptual contribution to the programme theory development.

Next, the literature will be examined by the research team for contexts, mechanisms and outcomes. Once identified, the data categorised according to context, mechanisms and outcomes will be recorded in a coding framework, and the coding will be managed using ATLAS. ti V.8.

\section{Step 5: data synthesis}

The data will be synthesised by organising the categorised C-M-Os into themes and subthemes prioritised by outcomes, thereby identifying patterns. We will use the recommended conceptual tools to test and adjust the programme theory ${ }^{34}$ :

- Juxtaposing: when reviewing data presented by the study to understand the outcome models mentioned in another paper.

- Reconciling: understanding why differences exist between contradictory sets of findings, which have occurred in seemingly similar situations.

- Adjudicating the data: judging the quality of research based on strengths and weaknesses in methodology.

- Consolidating sources of evidence: by developing a multidimensional reasoning for the intervention to explain why there are different outcomes in particular contexts.

- Situating: to explain the differing outcomes of intervention through the varying configurations of the context—mechanism-outcome.

\section{Phase 2: realist evaluation}

The initial part of our realist evaluation will be undertaken in tandem with the above realist synthesis and will facilitate the development of the initial programme theory. We will develop our initial programme theory by drawing on the research team's content expertise (being directly involved in implementing the programme across one key geographical area of NSW), considering the outcomes we anticipate occurring, and how we believe these might come about. As such, we will develop a practice-informed set of C-M-Os.

As more 'lines of enquiry' ${ }^{27}$ are identified, for example, through interviews with key stakeholders who have been involved in initiating and developing the Assistant in Medicine Programme, these will be followed up, and further literature searches will be conducted (dovetailing onto our realist synthesis). In this way, our realist syntheses and evaluation will be iterative by nature, allowing for an ongoing and complex interpretation of the programme theory and the development of middle-range theories (ie, specific hypotheses that can be tested empirically and have a transferable quality).$^{29}$

\section{Participants}

Stakeholders participating in the realist evaluation will comprise as below:

Programme developers $(n=15)$ are the members of NSW Health who originally devised the initiative, university representatives who have been involved in recruiting and advising on the implementation of the initiative and local health district representatives who are involved in developing the initiative on the ground.

Assistants in medicine ( $\mathrm{n}=20$ for interviews and audio diaries; $\mathrm{n}=55$ for questionnaires) comprise the final-year medical students employed by the specific local health district we are studying.

Assistant in medicine supervisors and team members ( $\mathrm{n}=40$ for interviews; $\mathrm{n}=200$ for questionnaires) comprise anyone who is working in the respective multidisciplinary team in which assistants in medicine are embedded. This includes interns, junior doctors, directors of medical services, junior medical officers (who the assistants in medicine will replace should the number of COVID-19 cases increase dramatically), junior medical officer managers, directors of assistant in medicine and interprofessional team members (eg, nursing and allied health).

\section{Data collection}

We will use a longitudinal, mixed-method approach to conduct the research over a period of 11 months (seven of which will comprise the data collection phase). The 
methods used will be interviews, surveys, and audio diaries (table 2).

Group (or, if requested, individual) interviews will be undertaken with all participant groups. Longitudinal audio diaries will be conducted with assistant in medicine participants to understand the lived experiences close to the events themselves. These will take around $10 \mathrm{~min}$ per week and comprise short narrative reflections on participants' ongoing experiences as an assistant in medicine with a focus on preparedness for practice, multidisciplinary team working and supervision. They will be provided with an audio diary guide prompting them what to record and instructions on where, when and how to send their recordings to the research team. They will be given an option to do the recordings on their own smartphone/tablet or on a digital voice recorder supplied by the research team.

We will also invite assistants in medicine to participate in a longitudinal questionnaire (administered twice over the course of their assistant in medicine employment) to assess their perceptions of professional identity, ${ }^{35}$ teamwork ${ }^{35}$ tolerance of uncertainty ${ }^{36}$ and burnout. ${ }^{37}$ Assistant in medicine supervisors and team members will also be asked to complete an online survey. The survey items will be developed based on the interview responses, and the rationale is to measure the prevalence of experiences that are narrated in the interviews across the participant cohort. Box 1 summarises the rationale for data collection for each participant group.

\section{Procedure}

Assistants in medicine, their supervisors and team members will be recruited from five locations within the local health district being studied (not identified for anonymity purposes). All recruitment and data collection with participant groups will take place by researchers electronically. Recruitment will commence as follows: the project officer will email participants to inform them of the study. The project officer will then forward the participant information sheet and consent form to those who express an interest in participating and organise timetables for sessions. For the assistants in medicine, they will also be introduced to the longitudinal audio diary part of the data collection, with the full details being explained to them during their interviews.

\section{Phase 3: analysis}

The C-M-O configurations of intended outcomes will be examined against the actual outcomes of the Assistants in Medicine initiative. Intended outcomes will comprise those developed during the realist syntheses as well as data from initial interviews with programme developers. Actual outcomes will be derived from our data collected from the assistants in medicine themselves and those who work with them. Thus, all data will be managed in a single ATLAS.ti V.8 database. Working with the C-M-O codes developed, we will compare and contrast the intended outcomes and associated mechanisms and contexts

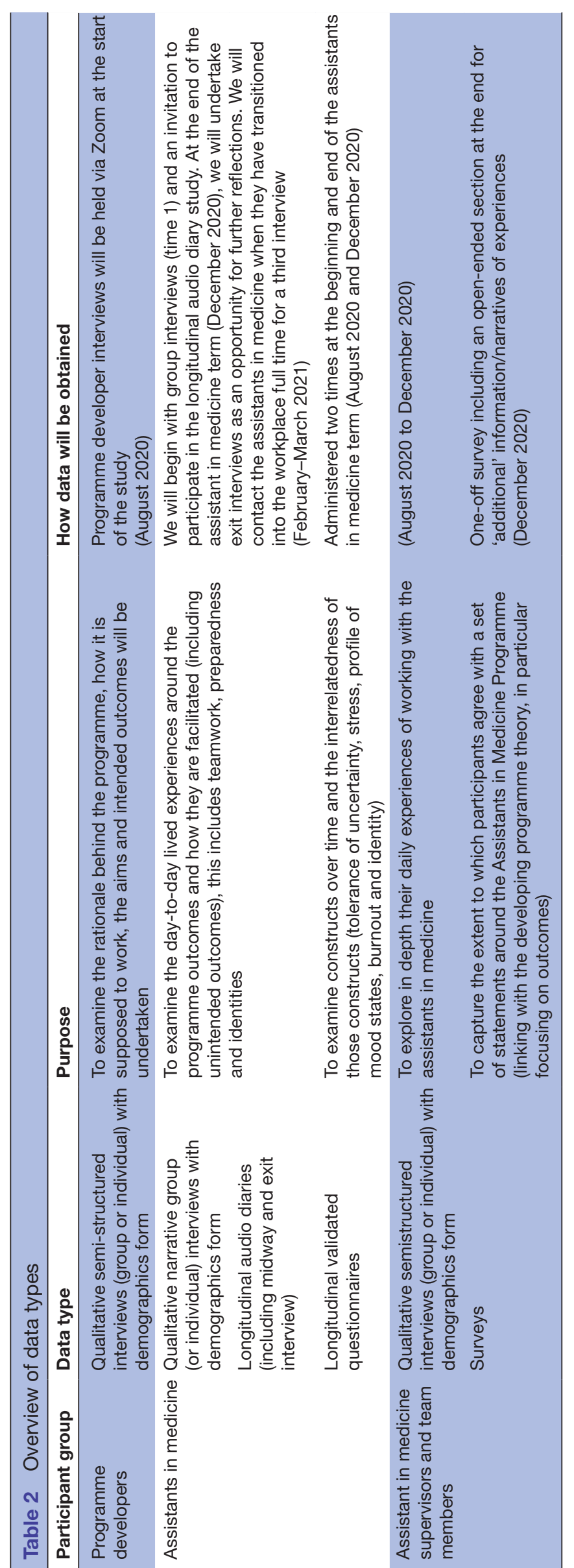


between the two sets of data (intended vs actual) to establish what worked for who, how and in what contexts. We will employ the process of data abduction. ${ }^{38}$ Abduction searches for an explanation of surprising results that are not readily explained by the initial programme theory. In doing so, we will consider new hypotheses or general rules that might explain any given case. This is an iterative process whereby hypotheses/rules are considered and data interrogated, until the expected results are discovered. Through this abductive process, we will formulate theoretical explanations based on empirical observations, drawing heavily on existing social theory as we consider the range of mediators for our explanations.

\section{Data analysis}

\section{Qualitative data (interviews and audio diaries)}

The audio recordings and transcripts will be loaded into a qualitative software (ATLAS.ti V.8) where they will be coded for data analysis. The use of ATLAS.ti V.8 will enable us to explore patterns across the data such as the similarities and differences in understandings and experiences across participant groups.

We will use a team-based primary-level analysis to identify outcomes $(\mathrm{O})$, mechanisms $(\mathrm{M})$ and contexts $(\mathrm{C})$ for the development of C-M-O configurations. ${ }^{27}$ These will be matched to the C-M-Os from the programme developers and realist synthesis (initial programme theory), refining them to ascertain the 'actual' programme theory.

In-depth narrative analysis of selected illustrative data sets will be conducted to shed further light onto our topic of inquiry, ${ }^{39-42}$ in particular focusing on the outcome of professional identities and preparedness, should we have sufficient resources.

\section{Quantitative data (demographics, questionnaires and surveys)}

We will analyse the numerical data (Likert Scales) using descriptive (eg, percentage, range and mean) and inferential (eg, t-tests and Analysis of Variance) approaches where possible. Descriptive analysis will enable us to determine the extent to which participants address the context, mechanisms and outcomes of the Assistant in Medicine Programme; inferential analyses will enable us to examine significant differences in opinions/experiences across participant groups and demographics. Appropriate non-parametric tests suitable for small sample analyses will be used should we receive fewer responses than expected. Other demographic categories may be added to or removed from this analysis of C-M-O configurations to test the developing theory. Open-ended questions will be analysed with the same team-based primary-level analysis as used for the qualitative data.

\section{Patient and public involvement statement}

Due to the tight timeframe, this study will be undertaken without patient and public involvement.

\section{Ethics and dissemination}

Ethical approval to undertake this study was granted by the Western Sydney Local Health District Human Research
Ethics Committee on 13 August 2020 (2020/ETH01745). The outcomes of this study will inform programme developers of the impact that the Assistants in Medicine initiative has on the workplace (ie, as identified in the outcomes of the C-M-O configurations). It will directly contribute to the development of the initial programme theory through an understanding of what actually happens. Our final report will be of interest to these programme developers: clinical schools, local health districts and policymakers in the NSW State Ministry of Health. It is envisaged that it will therefore affect future decision-making around the assistants in medicine role. We will publish our findings in peer-reviewed medical education journals and at international conferences.

Correction notice This article has been corrected since it was published. Given name of author 'Deborah 0'Mara' has now been spelled out.

Twitter Lynn V Monrouxe @LynnMonrouxe

Contributors Each named author has substantially contributed to the following: Conception: LVM and PH. Drafting the work: LM and JAD. Design of the work, revising it critically for important intellectual content and agreement to be accountable for all aspects of the work in ensuring that questions related to the accuracy or integrity of any part of the work are appropriately investigated and resolved: LVM, PH, PK, CK, LM, DAO'M, AR, ST and JAD.

Funding The authors have not declared a specific grant for this research from any funding agency in the public, commercial or not-for-profit sectors.

Competing interests None declared.

Patient and public involvement Patients and/or the public were not involved in the design, conduct, reporting or dissemination plans of this research.

Patient consent for publication Not required.

Provenance and peer review Not commissioned; externally peer reviewed.

Open access This is an open access article distributed in accordance with the Creative Commons Attribution Non Commercial (CC BY-NC 4.0) license, which permits others to distribute, remix, adapt, build upon this work non-commercially, and license their derivative works on different terms, provided the original work is properly cited, appropriate credit is given, any changes made indicated, and the use is non-commercial. See: http://creativecommons.org/licenses/by-nc/4.0/.

\section{ORCID iDs}

Lynn V Monrouxe http://orcid.org/0000-0002-4895-1812

Jennifer Ann Davids http://orcid.org/0000-0003-0861-9994

\section{REFERENCES}

1 Chen $\mathrm{HC}$, van den Broek WES, ten Cate O. The case for use of entrustable professional activities in undergraduate medical education. Acad Med 2015;90:431-6.

2 Crossley JG, Vivekananda-Schmidt P. Student assistantships: bridging the gap between student and doctor. Adv Med Educ Pract 2015;6:447-57.

3 Fullbrook A, Ross M, Mellanby E, et al. Initial experiences of a student assistantship. Clin Teach 2015;12:310-4.

4 Jones OM, Okeke C, Bullock A, et al. 'He's going to be a doctor in August': a narrative interview study of medical students' and their educators' experiences of aligned and misaligned assistantships. BMJ Open 2016;6:e011817.

5 Wells SE, Bullock A, Monrouxe LV. Newly qualified doctors' perceived effects of assistantship alignment with first post: a longitudinal questionnaire study. BMJ Open 2019;9:e023992.

6 Monrouxe LV, Bullock A, Tseng H-M, et al. Association of professional identity, gender, team understanding, anxiety and workplace learning alignment with burnout in junior doctors: a longitudinal cohort study. BMJ Open 2017;7:e017942.

7 Adamson TE. Critical issues in the use of physician associates and assistants. Am J Public Health 1971;61:1765-79.

8 Brown MEL, Laughey W, Tiffin PA, et al. Forging a new identity: a qualitative study exploring the experiences of UK-based physician associate students. BMJ Open 2020;10:e033450. 
9 Halter M, Wheeler C, Drennan VM, et al. Physician associates in England's hospitals: a survey of medical directors exploring current usage and factors affecting recruitment. Clin Med 2017;17:126-31.

10 Hoggins R, Scott-Smith W, Okorie M. Uk physician associate primary care placements: staff and student experiences and perceptions. Int $J$ Med Educ 2018;9:286-92.

11 Mahase E. Medical associates: junior doctors oppose professional equivalency. BMJ 2019;365:I2265.

12 Rimmer A. Medical associate professions: how physician associate and similar roles are developing, and what that means for doctors. BMJ 2018;362:k3897.

13 Roberts S, Howarth S, Millott H, et al. Experience of the impact of physician associates on -postgraduate medical training: A mixed methods -exploratory study. Clin Med 2019;19:4-10.

14 Williams LE, Ritsema TS. Satisfaction of doctors with the role of physician associates. Clin Med 2014;14:113-6.

15 Drennan V, Levenson R, Halter M, et al. Physician assistants in English general practice: a qualitative study of employers' viewpoints. J Health Serv Res Policy 2011;16:75-80.

16 Farmer J, Currie M, Hyman J, et al. Evaluation of physician assistants in national health service Scotland. Scott Med J 2011;56:130-4.

17 Hodgson H. A new kid on the block. Clin Med 2014;14:219-20.

18 Hooker RS, Hogan K, Leeker E. The globalization of the physician assistant profession. The Journal of Physician Assistant Education 2007;18:76-85.

19 Maoz-Breuer R, Berkowitz O, Nissanholtz-Gannot R. Integration of the first physician assistants into Israeli emergency departments - the physician assistants' perspective. Isr J Health Policy Res 2019;8:4.

20 White H, Round JEC. Introducing physician assistants into an intensive care unit: process, problems, impact and recommendations. Clin Med 2013;13:15-18.

21 Rees CE, Monrouxe LV. Medical students learning intimate examinations without valid consent: a multicentre study. Med Educ 2011;45:261-72

22 Feeney S, O'Brien K, O'Keeffe N, et al. Practise what you preach: health behaviours and stress among non-consultant Hospital doctors. Clin Med 2016;16:12-18.

23 Dornan T, Boshuizen H, King N, et al. Experience-Based learning: a model linking the processes and outcomes of medical students' workplace learning. Med Educ 2007;41:84-91.

24 Monrouxe L, Bullock A, Cole J. How prepared are UK medical graduates for practice: final report from a programme of research commissioned by the general medical Council. UK: General Medical Council, 2014.

25 Dornan T. Workplace learning. Perspect Med Educ 2012;1:15-23.
26 Drennan VM, Halter M, Wheeler C, et al. What is the contribution of physician associates in hospital care in England? a mixed methods, multiple case study. BMJ Open 2019;9:e027012.

27 IN E, Greenhalgh J, Manzano A. Realist research, guidelines, and the politics of evidence. London: Sage, 2018.

28 Pawson R, Greenhalgh T, Harvey G, et al. Realist review--a new method of systematic review designed for complex policy interventions. J Health Serv Res Policy 2005;10 Suppl 1:21-34.

29 Wong GW G, Pawson R, Greenhalgh T. Realist synthesis: rameses training material. London, 2013b: 1-54. https://www.ramesesproject. org/media/Realist_reviews_training_materials.pdf

30 Wong G, Greenhalgh T, Westhorp G, et al. RAMESES publication standards: realist syntheses. BMC Med 2013;11:21.

31 Babovic M, Fu R-H, Monrouxe LV. Understanding how to enhance efficacy and effectiveness of feedback via e-portfolio: a realist synthesis protocol. BMJ Open 2019;9:e029173.

32 Huang Y, Monrouxe LV, Huang C-D. The influence of narrative medicine on medical students' readiness for holistic care practice: a realist synthesis protocol. BMJ Open 2019;9:e029588-e88.

33 Wong G, Greenhalgh T, Westhorp G, et al. RAMESES publication standards: realist syntheses. BMC Med 2013;11:21.

34 RAMESES. The RAMESES projects: secondary the RAMESES projects, 2013. Available: https://www.ramesesproject.org/

35 Adams K, Hean S, Sturgis P, et al. Investigating the factors influencing professional identity of first-year health and social care students. Learning in Health and Social Care 2006;5:55-68.

36 Hancock J, Roberts M, Monrouxe L, et al. Medical student and junior doctors' tolerance of ambiguity: development of a new scale. Adv Health Sci Educ Theory Pract 2015;20:113-30.

37 Kristensen TS, Borritz M, Villadsen E, et al. The Copenhagen burnout inventory: a new tool for the assessment of burnout. Work \& Stress 2005;19:192-207.

38 Philipsen K. Theory building: using Abductive search strategies. Singapore: Springer Singapore, 2017: 45-71.

39 Jones OM, Okeke C, Bullock A, et al. 'He's going to be a doctor in August': a narrative interview study of medical students' and their educators' experiences of aligned and misaligned assistantships. BMJ Open 2016;6:e011817.

40 Monrouxe L, Sweeney K. Contesting narratives: medical professiona identity formation amidst changing values. Emerging Values in Health Care 2010:61-78.

41 Monrouxe L. Identities, self and medical education. In: Oxford textbook of medical education. Oxford: OUP Oxford, 2013: 113-23.

42 Monrouxe L, Rees C. Theoretical perspectives on identity: researching identities in healthcare. Researching medical education. 129. Hoboken: John Wiley \& Sons, 2015. 